\title{
A Prospective Study of Cholilithiasis in Children
}

\author{
Raiees Ahmad*, Alfer Nafae, Shahnawaz Bashir, Pervaze Salam, Qayoom Khan, \\ Malik Suhail, Umer Mushtaq, Javid Ahmad \\ Department of Surgery, Government Medical College, Srinagar, Jammu and Kashmir, India \\ Email: mallahraiees@gmail.com
}

Received 17 February 2015; accepted 24 March 2015; published 26 March 2015

Copyright @ 2015 by authors and Scientific Research Publishing Inc.

This work is licensed under the Creative Commons Attribution International License (CC BY). http://creativecommons.org/licenses/by/4.0/

c) (7) Open Access

\section{Abstract}

Background: Gallstones are generally uncommon in infants and children. Formation of gallstone is a very poorly understood phenomenon. In general, the risk factors for cholithiasis in infants include patients who are ill, are receiving hyperalimentation, are premature, have congenital anomalies and have necrotizing enterocolitis. Children aged 1 - 5 years most frequently have hemolysis as the underlying condition [1]. Materials and Methods: From Jan 2012 to Feb 2014, a study entitled “A Prospective Study of Cholelithiasis in Children" was conducted in Postgraduate Department of General Surgery Govt. Medical College Srinagar. The patients selected for the study were in the age group of 1 to 14 years of either sex. There were a total of 141 cases, out of which only 38 had ultrasound documented gallstones. All the patients included in the study were evaluated for prevalence, clinical presentation, and pathological features of gallstones, and were analysed for metabolic causes of gallstones. Results: The prevalence of chliothiasis in symptomatic patients was found to be $26.95 \%$ higher than the prevalence of gallstones in children in other parts of world, also the mean age of presentation was 9.3 years ranging from 6 - 14 years. Male to female ratio was 3:2 and male predominance was found in all age groups contrary to female predominance in adults. Most common presenting symptom was right upper quadrant pain followed by vomiting and nausea similar to presentation of symptomatic gallstones in adults. 4 patients had a positive family history of cholithiasis in the first degree relatives; 25 (65.7\%) patients had no underlying risk factor for gallstones contrary to presumption that gallstones in children are mostly secondary to some hematological disorder or other predisposing factors. Chronic cholecystities was found in $\mathbf{8 1 \%}$ of patients with gallstones and composition of gallstones retrieved was different from those of adult gallstones with calcium carbonate gallstones relatively common in children but composition of black and brown stones was almost similar to adult stones. Conclusion: Gallstone disease was increasingly gaining recognition in peadiatic practice due to significant documented increase in non-heamolytic cases over the last two decades. The observed gallstones prevalence, clinical presentation, pathological features of gallstones, and analysed metabolic causes of gallstones in children are different from those stones found in adults. The results of our

${ }^{*}$ Corresponding author.

How to cite this paper: Ahmad, R., Nafae, A., Bashir, S., Salam, P., Khan, Q., Suhail, M., Mushtaq, U. and Ahmad, J. (2015) A Prospective Study of Cholilithiasis in Children. Surgical Science, 6, 149-156. http://dx.doi.org/10.4236/ss.2015.63024 
study demonstrated that gallstones and gallstone related complications in peadiatic populations were different from the adult gallstone disease and there was an increase in prevalence of gallstones in children with no under lying risk factor for gallstones, and surgery remained the corne stone of treatment for peadiatric cholithiasis as in adults.

\section{Keywords}

\section{Peadiatric Cholelithiasis, Symptomatic Cholelithiasis, Peadiatric Age Group, Gallbladder Stones}

\section{Background}

Gallbladder calculi are relatively uncommon in children. However, the incidence of cholilithiasis has been increasing recently. Children may harbor cholesterol gallstones. Gallstones are generally uncommon in infants and children. The recently reported increasing number of cholecystectomies in this population refers mainly to girls aged over 16 years, obese, parous, and/or of Mexican-American origin [2]. All epidemiological studies showed that increasing age was associated with an increased prevalence of gallstones. Gallstones are four to ten times more frequent in older than younger subjects. Biliary cholesterol saturation increases with age, due to a decline in the activity of cholesterol $7 \alpha$ hydroxylase, the rate limiting enzyme for bile acid synthesis [3].

Moynihan's aphorism: a stone is a tombstone erected to the memory of the organism within it. Available literature establishes that various viable pathogenic bacteria that can be cultured in vitro are present in gallstones [4]-[6]. Scanning electron microscopy of fresh stones has revealed bacterial microcolonies on the gallstone surface as well as in the interior of pigmented gallstones [7]. More significantly, some studies have demonstrated viable bacteria present on gallstone which can be cultured in vitro. Escherichia coli, Klebsiella pneumonia, Pseudomonas aeruginosa and Enterococcus species were the commoner organisms grown from crushed gallstones.

Formation of gallstone is a very poorly understood phenomenon. Surprisingly, in the last few decades, there has been a significant rise in gallstone disease among children. Removal of the gallbladder by surgical method is the only solution available to the gallstone disease today and therefore, the disease has a strong impact on children [8].

The incidence of cholecystitis in the pediatric population varies mostly according to age, with some racial and cultural influences. In general, the risk factors for cholithiasis in infants include patients who are ill, are receiving hyperalimentation, are premature, have congenital anomalies and have necrotizing enterocolitis. Children aged 1 - 5 years most frequently have hemolysis as the underlying condition [1].

Cholelithiasis in infancy is often related to acute and chronic illness and hyperalimentatiion. Risk factors include abdominal surgery, sepsis, bronchopulmonary dysplasia, hemolytic disease, malabsorption, necrotizing enterocolitis and hepatobiliary disease. Other factors implicated include polycythemia, phototherapy and distal ileal resection. The immature hepatobiliary system of infants may predispose them to stone formation. Decreased hepatobiliary flow and immature bilirubin conjugation both contribute to stasis and sludge formation. Up to one half of infantile gallstones, especially those associated with hyperalimentation may resolve spontaneously [9].

Pregnancy, birth control pills, dehydration and obesity have contributory roles. Early menarche has been shown to significantly increase incidence, perhaps because of the lithogenic effect of estrogen on bile. Racial and genetic influences in the adolescent age group are similar to those in adults [10].

The present study has been undertaken to study prevalence, clinical presentation, and pathological features of gallstones, and to analyse metabolic causes of gallstones in children.

\section{Material and Methods}

From Jan 2012 to Feb 2014, a study entitled “A Prospective study of cholelithiasis in children” was conducted in Post Graduate Department of General Surgery Govt. Medical College Srinagar a prospective study and comprised of patients admitted with right upper quadrant abdominal pain in the various surgical wards of SMHS Hospital Srinagar. The patients selected for the study were in the age group of 1 to 14 years of either sex. The 
study was undertaken to study prevalence, clinical presentation, pathological features of gallstones, and to analyse metabolic causes of gallstones in children.

Subsequent to hospital admission, patients were evaluated on the basis of detailed history, thorough clinical examination and relevant investigations. Detailed history regarding the abdominal symptoms particularly the right upper quadrant pain was sought. Any associated history of nausea/vomiting, anorexia, hematuria and jaundice were recorded. History of any hemolytic disorder, hepatobiliary disease, abdominal surgery, artificial heart valves, pregnancy, obesity or bariatric surgery was noted. Exercise, drug history, diet, any past history of jaundice as well as menstrual history was recorded.

Detailed general physical examination and systemic examination was done.

All routine baseline investigations were done which included CBC, KFT, Electrolytes, LFT, Routine urine examination, Chest X-ray, X-ray abdomen and ECG. All patients entitled for the study were subjected to ultrasonography of abdomen and pelvis. The abdominal ultrasonography represented the most reliable and readily accessible study to detect gallstones and that it has been reported to have a success rate as high as $95 \%$. So ultrasonography was considered mandatory for the diagnosis of cholelithiasis in these patients.

Patients with suspected history of hemolytic disorder were subjected to detailed hemolytic profile. The lipid profile of these patients was done and stool examination for ova of Ascariasis Lumbricoides or any other parasitic infestation.

All patients who were diagnosed as having gallstones were categorized into two groups: A, patients with acute symptoms directly relevant to gallstones and B, those with nonspecific symptoms (incidental cholelithiasis). The patients with acute symptoms were initially subjected to conservative treatment with nothing per oral, IV fluids, antibiotics and antispasmodics. Those who did not respond to conservative treatment or developed complications were subjected to emergency cholecystectomy or cholecystostomy. Other patients were treated with elective cholecystectomy (laparoscopic or open).

Subsequently gallstones removed at the surgery were sent for stone analysis by chemical methods to determine its nature and composition.

Regular follow up of these patients was done two weekly for a period of two months and afterwards monthly for a period of four months to determine any subsequent complication or the resolution/recurrence of the symptoms.

\section{Results \& Observations}

There were a total of 141cases, out of which only 38 had ultrasound documented gallstones. As such, the prevalence rate of gallstone in our study was 26.95 .

The age range of patients in our study was 6 to 14 years, with the mean age of 9.3 years as shown below in Table 1.

There were 23 males (60.50) and 15 females (39.50) in our study. With regard to gender distribution as per the age group, there were 10 and 13 males within the age group of 6 - 10 and 11 - 14 years respectively. Similarly, there were 6 females in the age group of 6 - 10 years and 9 in the $11-14$ years age group, as depicted in Table 2 below.

Table 1. Age distribution of patients.

\begin{tabular}{ccc}
\hline Age range (years) & No. of patients & Percentage \\
\hline $1-5$ & 0 & 0 \\
$6-10$ & 16 & 42.10 \\
$11-14$ & 22 & 57.90 \\
\hline
\end{tabular}

Table 2. Age distribution of patients with relation to gender.

\begin{tabular}{ccccc}
\hline \multirow{2}{*}{ Age range (years) } & \multicolumn{3}{c}{ Males } & \multicolumn{2}{c}{ Females } \\
\cline { 2 - 5 } & No. & \% & No. & $\%$ \\
\hline $6-10$ & 10 & 26.30 & 6 & 15.70 \\
$11-14$ & 13 & 34.20 & 9 & 23.60 \\
\hline
\end{tabular}


Eleven (28.9\%) of the children belonged to "lower" class, 23 (60.50\%) children were of the "average" socioeconomic status and only $4(10.50 \%)$ children belonged to "upper" class, as represented in the below mentioned Table 3.

Among the group A patients, 34 (89.4\%) presented with RUQ pain, nausea, vomiting in 23 (60.5\%) 10 (26.3\%) had abdominal tenderness, 5 (13.1\%) had clinical jaundice and 3 (7.8\%) had a palpable lump referring to the RUQ. Fever was present in 2 (5.2\%) of the patients. These clinical parameters are represented in Table 4 with frequency.

A complete haemogram including hemoglobin level, total leucocyte count (TLC). Differential leucocytes count (DLC) and blood grouping was done routinely in all the patients. Peripheral blood film was normal in all the patients in our study as shown in Table 5.

There were 10 patients with blood group "A", 8 patients with "B”, 7 with group "AB", and 13 patients belonging to blood group "O" as shown in below Table 6 .

Nine patients in our study had raised levels of Bilirubin, 6 patients had significantly raised levels of alkaline phosphatase and 4 patients had raised levels of transferases. These results are shown in Table 7.

Table 3. Distribution of patients as per the socioeconomic status.

\begin{tabular}{ccc}
\hline Upper class & Average class & Lower class \\
\hline 4 & 65 & 11 \\
\hline Table 4. Grouping of patients as per the clinical presentation. \\
\hline & No. of patients & Percentage (\%) \\
\hline RUQ & 34 & 39.4 \\
Nausea/vomiting & 23 & 60.5 \\
Abdominal tenderness & 10 & 26.3 \\
Jaundice & 5 & 13.1 \\
Palpable lump & 3 & 7.8 \\
Fever & 2 & 5.2 \\
\hline
\end{tabular}

Table 5. Showing haemogram of the patients.

\begin{tabular}{ccc}
\hline & Normal haemogram & Abnormal haemogram \\
\hline No. of patients & 25 & 13 \\
Percentage & 65.7 & 34.2 \\
\hline
\end{tabular}

Table 6. Blood grouping of patients.

\begin{tabular}{ccc}
\hline Blood group & No. of patients & Percentage \\
\hline A & 10 & 26.3 \\
B & 8 & 21.0 \\
AB & 7 & 18.4 \\
O & 13 & 34.2 \\
\hline
\end{tabular}

Table 7. The results of the liver function tests.

\begin{tabular}{cccc}
\hline Blood group & $\begin{array}{c}\text { Bilirubin } \\
\text { (no. of patients) }\end{array}$ & $\begin{array}{c}\text { Alkaline } \\
\text { phosphates }\end{array}$ & $\begin{array}{c}\text { Transferases } \\
\text { (no. of patients) }\end{array}$ \\
\hline Raised levels & 9 & 7 & 4 \\
Normal levels & 29 & 31 & 34 \\
\hline
\end{tabular}


The triglycerides levels were raised in 7 patients (22.5\%) and total cholesterol was raised in 1 patient (2.6\%) as shown in Table 8.

Out of the total no. of 38 patients, 14 (36.84\%) had multiple gallstones while the rest of the patients had a single stone in the gall bladder. Only one patients had associated CBD stone and 13 (34.2\%) had contracted thick walled gall bladder. 32 (84.2\%) of our patients had stone size of less than $5 \mathrm{~mm}$ while the rest of the six patients has a stone size of more than $5 \mathrm{~mm}$. Ultrasonographic features are depicted in Table 9 below.

All the patients who presented with acute symptoms and signs were subjected to conservative treatment with NPO, IV fluids, antibiotics and antispasmodics. In our study, 18 (47.3\%) patients presented with acute symptoms and the rest had non specific symptoms either vague abdominal pains, colics, food intolerance etc. Out of these 18 patients who presented with the acute symptoms all but one settled with the above treatment and were subsequently subjected to elective cholecystectomy afterwards. Emergency cholecystectomy was done in one patient who did not settle with the conservative treatment. Rest of the patients were treated by elective cholecystectomy, either by open or laparoscopic method. Results are shown in Table 10 below.

\section{Discussion}

Gallstone disease which has long been considered an adult disease, is increasingly gaining recognition in peadiatric practice because of significant documented increase in non-hemolytic cases. Gallstones are mostly an incidental finding in children but should be strongly considered in workup of those with non specific abdominal pain with associated risk factors.

The prevalence rate of gallstones in our study was found to be $26.95 \%$. Acalovschi M et al. [11] found that the median prevalence rate ranges from $5.9 \%$ to $21.9 \%$ with highest rate seen in Bergen, Norway and Schwedt, Germany and the lowest in Chianciacano and Sirmione, Italy.

The age range of patients in our study was 6 - 14 years with a mean age of 9.3 years. In a study conducted by Kumar R et al. [12] median age at presentation was 10 years. In studies conducted by S. Reif et al. [13] the mean age was 12.2 years.

In our study, 16 patients (42.1\%) were in age group 6 - 10 and 22 patients (57.8\%) in age group of 11 - 14 years. There was no patient in the age group of $1-5$ in our study. Thus maximum number of cases was found in the age group of 11 - 14 years. Friesen et al. [14] reported in a review of 693 children with gallstones that infants less than 6 months of age represented 10\% of cases, 69\% were in age group of 11 - 21 years and 21\%

Table 8. Lipid profile.

\begin{tabular}{cccccc}
\hline & TC & HDL & LDL & VLDL & TG \\
\hline Raised & 1 & 0 & 0 & 0 & 7 \\
Normal & 37 & 38 & 38 & 38 & 31 \\
Percentage of raised & 2.6 & 0 & 0 & 0 & 22.5 \\
\hline
\end{tabular}

Table 9. Ultrasonographic characteristics of the gallstones.

\begin{tabular}{|c|c|c|c|c|c|c|c|c|}
\hline & \multicolumn{2}{|c|}{ Gallstones } & \multicolumn{2}{|c|}{ Associated CBD stones } & \multicolumn{2}{|c|}{ Wall thickness } & \multicolumn{2}{|c|}{ Stone size } \\
\hline & Single & Multiple & Present & Absent & Normal & Increased & $<5$ & $>5$ \\
\hline No. of patients & 24 & 14 & 1 & 37 & 25 & 13 & 32 & 6 \\
\hline Percentage & 63.15 & 36.84 & 2.6 & 97.3 & 65.7 & 34.2 & 84.2 & 15.7 \\
\hline
\end{tabular}

Table 10. Operative treatment.

\begin{tabular}{ccccc}
\hline & \multicolumn{2}{c}{ Emergency treatment } & \multicolumn{2}{c}{ Elective treatment } \\
\cline { 2 - 5 } & Cholecystostomy & Cholecystostomy & Open cholecystectomy & Lap. cholecystectomy \\
\hline No. of patients & 1 & Nil & 32 & 84.2 \\
Percentage & 2.6 & - & 15.7 \\
\hline
\end{tabular}


between 6 months and 10 years. In the US, the reported prevalence of gallstones in children is $0.15 \%$ to $0.22 \%$, where as in adults it is $4 \%-11 \%$ [12].

The number of male patients our study was 23 and females were 15 in number. The male to female ratio was 3:2 and this male predominance was found in all age groups. Similar results were found in studies conducted by Kumar R et al. [12] as well by Mohmmad A. Bakhotmah [15].

Diehl et al. [16] analysed the socioeconomic status in Mexican Americans as compared with non-Hispanic Whites and observed that those living in affluent neighbourhoods, with high occupational status, high family incomes and high educational attainment had lower rates of gallstones than those lower on social scale. In our study $11(28.9 \%)$ children belonged to lower class, 23 (60.5\%) children were of the average socioeconomic status and only 4 (10.5\%) were of the upper class. In our study 4 patients had positive family history of cholithiasis in first degree relatives. Aharon Klar et al. [17] in their study had $7.5 \%$ of their patients with family history of cholithiasis.

The clinical presentation of patients in our study was as follows in decreasing order of frequency.

- Right upper quadrent pain $\rightarrow 34$ (89.4\%).

- Nausea/vomiting $\rightarrow 23$ (60.5\%).

- Jaundice $\rightarrow 5$ patients (13.1\%).

- Lump palpable in right upper quadrant $\rightarrow 3(7.8 \%)$.

- Asymptomatic $\rightarrow 4$ (10.5\%). These patients had abdominal ultrasound done for some other disease and were diagonosede as having coincidental cholithiasis.

In a study conducted by Pollak R et al. [18] found that most of the presenting symptom of pediatric cholithiasis was vague right upper quadrant and epigastric pain (95\%). However Craig A found that, overall most common symptom in their series was vomiting (60\%) [14].

Peaditric cholithiasis was viewed as disease of prematurity, usually related to the use of total parental nutrition or in adolescence, usually to hemolytic disease. In our series only three children had heamolytic disease (97.8\%) and 25 patients (5.7\%) had no under lying illness or risk factor. In a study conducted by Kumar Rajendra et al. [12] 102 cases of cholithiasis in children and found no cause in 66 patients (64\%). Comparable results were found in our study.

The treatment of choice for cholithiasis is cholecystectomy. Willis A et al. found that prognosis for recovery is excellent for peadiatic cholithiasis after cholecystectomy if there is no complicating systemic disease [2]. In our study 84.2\% patients (32) underwent open holecystectomy as compared to 6 patients (15.7\%) had cholecystectomy done by laparoscopic method.

Histopatholgical examination of removed gallbladder showed features of chronic cholecystities in 31 patients (81\%), congested gall bladder in 4 patients (10.5\%) and fibrotic inflamed gall bladder in 3 patients (7.8\%). In a study conducted by Bakhotmah A, features of chronic cholecystities were found in 75 of patients were as congested gall bladder and fibrotic inflamed gall bladder in $12.5 \%$ each [15].

All the stones retrieved during surgery were sent for analysis by chemical methods. We found that 18 patients (47.3\%) had cholesterol stones, 10 patients (26.33\%) had stones composed of calcium bilrubinate and 8 patients (21\%) had mixed stones. Calcium carbonate stones were found in only 2 (5.2\%) of our patients. Analysis suggests that the composition of black and brown stones from both children and adults are similar. Black stones from adults and children are rich in bilrubin. Brown stones are composed of varying amounts of bilrubin and cholesterol. In contrast to adults calcium carbonate gallstones are relatively common in children [19].

Stringer MD et al. [20] performed composition studies of gallstones by Fourier transform infrared microspectroscopy. Of the 20 children, 10 (50\%) had black pigment stones, 2 (10\%) had cholesterol stones, 1 (5\%) had brown pigment stones and 7 (35\%) had calcium carbonate stones; later are exceptionally rare in adults.

There were no major complications of surgery in all of our patients; except for minor wound infection in two of the patients which delayed their early discharge from hospital. All the patients were discharged home after 3 9 days postoperatively, with an average in hospital stay of 3.38 days postoperatively.

All the patients in our study were on regular follow up. We found no patients with recurrence of symptoms or with retained stones.

\section{Conclusion}

Cholilithiasis in peadiatic age groups is relatively uncommon but can affect any age and sex. Gallstone disease 
was increasingly gaining recognition in peadiatic practice due to significant documented increase in non-heamolytic cases over the last two decades. The observed gallstones prevalence, clinical presentation, pathological features of gallstones, and analysed metabolic causes of gallstones in children are different from those stones found in adults. Gallstones are most commonly an incidental finding in children. The prevalence of chliothiasis in symptomatic patients was found to be $26.95 \%$ higher than the prevalence of gallstones in children in other parts of world, and the mean age of presentation was 9.3 years ranging from 6 - 14 years. Male to female ratio was 3:2 and male predominance was found in all age groups contrary to female predominance in adults. Most common presenting symptom reported was right upper quadrant pain (89.4\%) and second most common presentation was nausea and vomiting (60.5\%), similar to presentation of symptomatic gall stones in adults. Among 38 patients, 4 (10.5\%) patients had positive family history of gall stones in first degree relatives. 25 (65.7\%) patients had no underlying risk factor for gall stones contrary to presumption that gall stones in children are mostly to some heamotological disorder or other predisposing factors. Chronic cholecystitis was found in $81 \%$ of patients with gallstones and composition of gallstones retrieved was different from those of adult gallstones with calcium carbonate gallstones relatively common in children but composition of black and brown stones were almost similar to adult stones. The above results demonstrated that gallstones and gallstone related complications in peadiatic populations was different from the adult gallstone disease and there was an increase in prevalence of gallstones in children with no under lying risk factor for gallstones.

\section{References}

[1] Holcomb Jr., G.W. and O. Neill Jr., J.A. (1980) Cholecystities, Cholelithiasis and Common Duct Stenosis in Children and Adults. Annals of Surgery, 191, 626-635. http://dx.doi.org/10.1097/00000658-198005000-00015

[2] Wingert, W.A. and Mikity, V.G. (1967) Cholithiasis and Cholecystities in Childhood. California Medicine, 107, 27-32.

[3] Danzinger, R.G., Gordon, H., Schoenfield, L.J., et al. (1972) Lithogenic Bile in Siblings of Young Women with Cholithiasis. Mayo Clinic Proceedings, 47, 762-766.

[4] Crichlow, R.W., Seltzer, M.H. and Jannetta, P.J. (1972) Cholecystitis in Adolescents. American Journal of Digestive Diseases, 17, 68-72. http://dx.doi.org/10.1007/BF02239263

[5] Van der Linden, W. and Simosan, N. (1973) Familial Occurrence of Gall Stone Disease. Incidence in Parents of Young Patients. Human Heredity, 23, 123-127. http://dx.doi.org/10.1159/000152563

[6] Andrassy, R.J. and Treadwell, T.A. (1976) Gall Bladder Disease in Children and Adolescents. American Journal of Surgery, 132, 19-21. http://dx.doi.org/10.1016/0002-9610(76)90282-8

[7] Ahlberg, J. (1979) Serum Lipid Levels and Hyperlipoprotinaemia in Gallstone Patients. Acta Chirurgica Scandinavica, 145, 373-377.

[8] Chirkow, G.T. and Highman, L.M. (1980) Cholelithiasis and Cholecystitis in Children after Repair of Congenital Duodenal Anomalies. Archives of Surgery, 115, 1.

[9] Fisher, M. and Rosenstein, J.J. (1981) Gall Bladder Disease in Children and Adolescents. Journal of Adolescent Health Care, 1, 309-312. http://dx.doi.org/10.1016/S0197-0070(81)80011-3

[10] Petitti, D., Friedman, G.D. and Klastky, A.L. (1981) Association of History of Gall Bladder Disease with a Reduced Concentration of High Density-Lipoprotein Cholesterol. New England Journal of Medicine, 304, 1396-1398. http://dx.doi.org/10.1056/NEJM198106043042305

[11] Acalovschi, M. (2001) Cholesterol Gallstones: From Epidemiology to Prevention. Postgraduate Medical Journal, 77, 221-229. http://dx.doi.org/10.1136/pmj.77.906.221

[12] Kumar, R., Nguyen, K. and Shun, A. (2001) Gallstones and Common Bile Duct Calculi in Infancy and Childhood. Australian and New Zealand Journal of Surgery, 70, 188-191. http://dx.doi.org/10.1046/j.1440-1622.2000.01783.x

[13] Reif, S., Sloven, D.G. and Lebenthal, E. (1991) Gallstones in Children. Characterization by Age, Etiology, and Outcome. American Journal of Diseases of Children, 145, 105-108.

[14] Friesen, C.A. and Roberts, C.C. (1989) Clinical Characteristics in Children: Case Analysis and Literature Review. Clinical Pediatrics, 28, 294-298. http://dx.doi.org/10.1177/000992288902800701

[15] Mohmad, B.A. (1999) Symptomatic Cholithiasis in Children: A Based Review. Saudi Journal of Gastroenterology, 12, $1-8$.

[16] Diehl, A.K., Rosenthal, M., Hazuda, H.P., Comeaux, P.J. and Stern, M.P. (1985) Socioeconomic Status and the Prevalence of Clinical Gallbladder Disease. Journal of Chronic Diseases, 38, 1019-1026. 
http://dx.doi.org/10.1016/0021-9681(85)90100-6

[17] Klar, A., Branski, D., Akerman, Y., Nadjari, M., Berkun, Y., Moise, J., et al. (2005) Sludge Ball, Pseudolithiasis, Cholelithiasis and Choledocholithiasis from Intrauterine Life to 2 Years: A 13 Year Follow-Up. Journal of Pediatric Gastroenterology \& Nutrition, 40, 477-480. http://dx.doi.org/10.1097/01.MPG.0000151749.01631.21

[18] Pollak, R., Reyes, H.M. and Pringle, K.C. (1982) Cholelitiasis in Childhood and Adolescence: A Review. South African Medical Journal, 62, 887-889.

[19] Schafmaye, C., Hartle, J., Tepe, J., Alber, S., Freitag, S., Völzke, H., et al. (2006) Predicators of Gallstone Composition in 1025 Symptommatic Gall Stones from Northern Germany. BMC Gastroenterology, 6, 36. http://dx.doi.org/10.1186/1471-230X-6-36

[20] Stringer, M.D., Taylor, D.R. and Solway, R.D. (2003) Gallstone Composition: Are Children Different. Journal of Pediatrics, 142, 435-440. http://dx.doi.org/10.1067/mpd.2003.159 